\title{
THE SOLUTION OF POSITIVE-SYMMETRIC HYPERBOLIC SYSTEMS BY DIFFERENCE METHODS
}

\author{
MILTON LEES ${ }^{1,2}$
}

1. Introduction. Consider the linear system of differential equations

$$
\sum_{i=1}^{n} A^{i}(x) \frac{\partial u}{\partial x_{i}}+B(x) u=f(x)
$$

where $u$ and $f$ are $m$-vector-valued functions, $A^{i}$ and $B$ are $m \times m$ matrix-valued functions, and $x=\left(x_{1}, x_{2}, \cdots, x_{n}\right)$ varies over all points of the real Euclidean $n$-space $E^{n}$ which have non-negative coordinates.

The system (1.1) is called positive-symmetric hyperbolic if the matrices $A^{i}$ are symmetric and positive-definite. Such systems are included in the general class of symmetric hyperbolic systems introduced by Friedrichs [1]. Symmetric systems are of particular interest because they possess an energy inequality; that is, the square integral of the solution over a space-like hyperplane can be estimated in terms of square integrals of initial values of the solution and the nonhomogeneous term in the differential equation.

We shall not consider the Cauchy problem for (1.1), but rather, we prescribe the initial values on the coordinate hyperplanes $x_{i}=0$, i.e.,

$$
u\left(x_{1}, \cdots, x_{i-1}, 0, x_{i+1}, \cdots, x_{n}\right)=g^{i}\left(x_{1}, \cdots, x_{i-1}, x_{i+1}, \cdots, x_{n}\right)
$$

for $i=1,2, \cdots, n$. That the initial value problem (1.1), (1.2) also possesses an energy inequality was proved by Wendroff [3]. The proof is similar to the one for the Cauchy problem given by Friedrichs.

Moreover, Wendroff demonstrated that a finite-difference analogue of (1.1), (1.2), having centered difference quotients, also satisfies an energy type inequality; the square integral of certain averages of the solution of the difference equation are estimated in terms of square integrals of averages of the initial data and $f$. Under the assumption that a genuine solution of (1.1), (1.2) exists, he proved, for $n>2$, that the finite-difference equation has a unique solution approximat-

Received by the editors May 23, 1960.

${ }^{1}$ Part of this work was done while the author was at the Brookhaven National Laboratory, Upton, L. I., New York.

2 This research was partially supported by the United States Air Force through the Air Force Office of Scientific Research of the Air Research and Development Command under contract No. AF49(638)-253. Reproduction in whole or in part is permitted for any purpose of the United States Government. 
ing, with arbitrary accuracy, the solution of (1.1), (1.2). The error is measured in terms of the norm induced by the energy estimate. When $n=2$ he was able to obtain pointwise estimates for the error.

In this paper, we shall construct the solution of (1.1), (1.2) by employing a finite-difference analogue of it which utilizes backward difference quotients. This backward difference analogue of (1.1), (1.2) also possesses an energy inequality from which we prove an approximation theorem similar to that in [3], but in our case the error is measured in the metric of $L^{2}$.

Existence and uniqueness will not be presupposed in this paper, but will be established as a consequence of the energy inequality for the backward difference equation. Existence and uniqueness will be proved under the assumption that the data for (1.1), (1.2) have a sufficient number of bounded derivatives. As a corollary of the existence theorem we prove that the finite-difference approximations to the solution of (1.1), (1.2) converge uniformly on compact sets.

In [3], the initial value problem was also investigated for the semilinear equation

$$
\sum_{i=1}^{n} A^{i}(x) \frac{\partial u}{\partial x_{i}}=F(x, u) .
$$

Our results and methods, with slight modifications, apply to $(1,3)$, and this will be briefly indicated. In addition, certain questions concerning (1.3) which have no counterpart in the linear case will also be discussed.

2. Preliminaries. Let $Q=\left\{x \in E^{n} \mid x_{i}>0, i=1,2, \cdots, n\right\}$, and let $\bar{Q}$ be the closure of $Q$. Set

$$
Q(x)=\left\{y \in E^{n} \mid 0 \leqq y_{i} \leqq x_{i}, i=1,2, \cdots, n\right\}
$$

and

$$
Q(x|i| a)=\left\{y \in E^{n} \mid 0 \leqq y_{j} \leqq x_{j} \text { for } i \neq j \text { and } y_{i}=a\right\} .
$$

Note that the sets $Q(x|i| 0)$ and $Q\left(x|i| x_{i}\right)$ make up the boundary of $Q(x)$.

We denote by $(u, v)$ the Euclidean scalar product of the two $m$ vectors $u$ and $v$, and we let $|u|=[(u, u)]^{1 / 2}$ be the associated norm. For a matrix $B$ we define $|B|$ to be the operator norm

$$
|B|=\sup _{u \neq 0} \frac{|B u|}{|u|} .
$$

Let $h$ be an arbitrarily chosen positive number, the mesh size. The 
points of $\bar{Q}$ whose coordinates are all non-negative integral multiples of $h$ are called mesh points, ${ }^{3}$ and the set of all mesh points will be denoted by $\bar{Q}_{h}$. Put $Q_{h}=Q \cap \bar{Q}_{h}$.

We define the translation operators $E_{i}$ and $\bar{E}_{i}$ as follows:

$$
\begin{aligned}
& E_{i}[v]\left(x_{1}, \cdots, x_{n}\right)=v\left(x_{1}, \cdots, x_{i}+h, \cdots, x_{n}\right), \\
& \bar{E}_{i}[v]\left(x_{1}, \cdots, x_{n}\right)=v\left(x_{1}, \cdots, x_{i}-h, \cdots, x_{n}\right) .
\end{aligned}
$$

In terms of these basic operators we define the forward and backward difference quotients as follows:

$$
\begin{aligned}
& v_{x_{i}}=h^{-1}\left\{E_{i}[v]-v\right\}, \\
& v_{\bar{x}_{i}}=h^{-1}\left\{v-\bar{E}_{i}[v]\right\}=E_{i}\left[v_{x_{i}}\right] .
\end{aligned}
$$

Difference quotients of higher order are formed in the obvious way. Let

$$
Q_{h}(x)=\text { all mesh points in } Q(x),
$$

and

$$
Q_{h}^{0}(x)=\text { all mesh points in } Q(x)-\bigcup_{i=1}^{n} Q(x|i| 0) .
$$

We turn now to a finite-difference problem similar to the problem (1.1), (1.2). We seek a function $v$ on the mesh $\bar{Q}_{h}$ which satisfies the backward difference equation

$$
\sum_{i=1}^{n} A^{i}(x) v_{\bar{x}_{i}}+B(x) v=f(x)
$$

in $Q_{h}$ and takes on the initial values

$$
v=g^{i}
$$

on $Q_{h}(\infty|i| 0), i=1,2, \cdots, n$.

We conclude this section with a sufficient condition for the finitedifference problem to have a unique solution.

Let $y \in Q_{h}$. Since the matrices $A^{i}(x)$ are positive-definite, there exist positive scalars $a^{i}(y)$ such that

$$
\left(A^{i}(x) \xi, \xi\right) \geqq a^{i}(y)|\xi|^{2}
$$

for $x \in Q_{k}(y)$, where $\xi$ is a real $m$-vector. Set

$$
|B|_{y}=\sup _{\boldsymbol{x} \in Q_{\boldsymbol{h}}(y) .}|B| \text {. }
$$

We could just as well have taken unequal mesh sizes along the different coordinate axes, but in the interests of simplicity we have elected to use a common mesh size. 
TheOREM 1. Let $y \in Q_{h}$. If the mesh size $h$ satisfies the condition

$$
h|B|_{\nu}<\sum_{i=1}^{n} a^{i}(y)
$$

then there exists a unique solution of the initial value problem (2.1), (2.2) in $Q_{h}(y)$.

Proof. We first construct the solution at the point $(h, h, \cdots, h)$. Once this is done, we can proceed, using the same construction, from point to point, until $Q_{h}(y)$ is exhausted.

Consider (2.1) at the point $(h, h, \cdots, h)$; it can be written in the form

$$
\left(B+\sum_{i=1}^{n} A^{i} h^{-1}\right) v=h^{-1} \sum_{i=1}^{n} A^{i} \bar{E}_{i}[v]+f .
$$

Since the terms $\bar{E}_{i}[v]$ are known, our problem now is to show that the matrix $C=B+h^{-1} \sum_{i=1}^{n} A^{i}$ is nonsingular. If $\xi$ is any real $m$ vector, then we have, in view of (2.3),

$$
(C \xi, \xi) \geqq\left[h^{-1} \sum_{i=1}^{n} a^{i}(y)-|B|_{y}\right]|\xi|^{2} .
$$

Because of (2.4) and (2.5), $(C \xi, \xi)>0$ for $|\xi| \neq 0$, and this implies that $C$ is nonsingular. It is obvious that the solution is unique.

In the case of the semi-linear equation, the situation is slightly more complicated. But if we assume that $F(x, u)$ satisfies a Lipschitz condition of the form

$$
|F(x, u)-F(x, v)| \leqq K(y)|u-v|, \quad x \in Q_{h}(y),
$$

then Theorem 1 holds for the semi-linear equation, provided that we add the condition

$$
h K(y)<\sum_{i=1}^{n} a^{i}(y)-|B|_{y} h
$$

on the mesh size $h$.

We sketch a proof of this fact. To find $v$ at the point $(h, h, \cdots, h)$, we set up the following iteration procedure:

$$
C v^{(k+1)}=\sum_{i=1}^{n} A^{i} h^{-1} \bar{E}_{i}[v]+F\left(v^{(k)}\right) .
$$

Because of Theorem 1, the iteration scheme is well defined. It follows from (2.5) that 


$$
\left|C^{-1}\right|_{y} \leqq h\left(\sum_{i=1}^{n} a^{i}(y)-h|B|_{y}\right)^{-1}
$$

and hence

$$
\left|v^{(k+1)}-v^{(k)}\right| \leqq\left|C^{-1}\right|_{y}\left|F\left(v^{(k)}\right)-F\left(v^{(k-1)}\right)\right| .
$$

Taking into account (2.6) and (2.7), we find that

$$
\left|v^{(k+1)}-v^{(k)}\right| \leqq \rho\left|v^{(k)}-v^{(k-1)}\right|,
$$

where $0<\rho<1$. Thus the sequence $\left\{v^{(k)}\right\}$ converges to a limit, say $v$, and $v$ clearly satisfies

$$
C v=\sum_{i=1}^{n} A^{i} h^{-1} \bar{E}_{i}[v]+F(v) .
$$

Again the solution is unique.

3. The energy inequality. Let $x \in Q_{h}$ and set

$$
\begin{aligned}
\|v\|_{Q(x)}^{2} & =h^{n} \sum_{y \in Q_{h}(x)}|v(y)|^{2}, \\
\|v\|_{Q^{0}(x)}^{2} & =h^{n} \sum_{y \in Q_{h}^{0}(x)}|v(y)|^{2}, \\
\|v\|_{Q(x|i| a)}^{2} & =h^{n-1} \sum_{y \in Q_{h}(x|i| a)}|v(y)|^{2} .
\end{aligned}
$$

THEOREM 2. Let $x \in Q_{h}$ and let $A^{i}$ be of class $C^{1}[Q(x)]$. If $v$ is a solution of the finite-difference problem (2.1), (2.2), then there exist constants $h_{0}>0$ and $C\left(h_{0}\right)>0$, depending only on $A^{i}, A_{x_{i}}^{i}, B, n$ and $x$, such that

$$
\sum_{i=1}^{n}\|v\|_{Q\left(x|i| x_{i}\right)}^{2} \leqq C\left(h_{0}\right) \sum_{i=1}^{n}\|v\|_{Q(x|i| 0)}^{2}+C\left(h_{0}\right)\|f\|_{Q^{0}(x)}^{2}
$$

for all $h \leqq h_{0}$.

Proof. Using the symmetry of the matrices $A^{i}$, we have

$$
\left(v, A^{i} v_{\bar{x}_{i}}\right)=\left(A^{i} v, v_{\bar{x}_{i}}\right)=\sum_{k=1}^{m}\left(A^{i} v\right)^{k} v_{\bar{x}_{i}}^{k}
$$

where $w^{k}$ denotes, in general, the $k$ th component of the $m$-vector $w$. It is readily verified that

$$
\sum_{k=1}^{m}\left[\left(A^{i} v\right)^{k} v^{k}\right]_{\bar{x}_{i}}=\left(A^{i} v, v_{\tilde{x}_{i}}\right)+\sum_{k=1}^{m}\left(A^{i} v\right)_{\bar{x}_{i}}^{k} \bar{E}_{i}\left[v^{k}\right]
$$

For the moment, let $\left\{b^{k_{s}}\right\}$ denote the elements of $A^{i}$. Then 


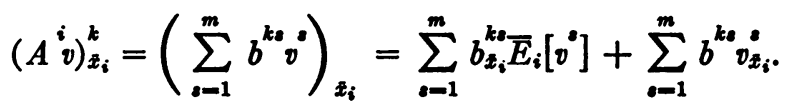

If we combine (3.2), (3.3) and (3.4), we obtain

$$
\left(v, A^{i} v_{\bar{x}_{i}}\right)=\left(v, A^{i} v\right)_{x_{i}}-\left(\bar{E}_{i}[v], A^{i} v_{x_{i}}\right)-\bar{E}_{i}\left(v, A_{x_{i}}^{i} v\right) .
$$

Now if we add the term $\left(v, A \boldsymbol{i}_{t_{i}}\right)$ to both sides of (3.5), and rearrange the result slightly, we obtain the basic identity

$$
2\left(v, A^{i} v_{x_{i}}\right)=\left(v, A^{i} v_{x_{i}}+h\left(v_{x_{i}}, A^{i} v_{x_{i}}\right)-\bar{E}_{i}\left(v, A_{x_{i}}^{i} v\right)\right. \text {. }
$$

Since the $A^{i}$ are positive-definite,

$$
\left(v_{\tilde{x}_{i}}, A^{i_{x_{x_{i}}}}\right) \geqq 0 .
$$

Taking the scalar product of equation (2.1) with $2 h^{n} v$, summing the result over the mesh points of $Q_{h}^{0}(x)$ rnd utilizing (3.6) and (3.7), we get

$$
\begin{aligned}
\sum_{i=1}^{n} h^{n} \sum_{Q_{h}^{0}(x)}\left(v, A^{i} v\right)_{x_{i}} \leqq & \sum_{i=1}^{n} h^{n} \sum_{Q_{h}^{0}(x)} \bar{E}_{i}\left(v, A_{x_{i}}^{i} v\right) \\
& +2 h^{n} \sum_{Q_{h}^{0}(x)}\{(v, B v)+(v, f)\} .
\end{aligned}
$$

Since

$$
\begin{aligned}
& (v, B v) \leqq|B|_{y}|v|^{2}, \\
& 2(v, f) \leqq|v|^{2}+|f|^{2},
\end{aligned}
$$

and

$$
h^{n} \sum_{Q_{h}^{0}(x)}\left(v, A^{i} v\right)_{x_{i}}=h^{n-1} \sum_{Q_{h}\left(x|i| x_{i}\right)}\left(v, A^{i} v\right)-h^{n-1} \sum_{Q_{h}(x|i| 0)}\left(v, A^{i} v\right)
$$

it follows from (3.8) that

$$
\text { (3.9) } \quad \sum_{i=1}^{n}\|v\|_{Q\left(x|i| x_{i}\right)}^{2} \leqq C_{1} \sum_{i=1}^{n}\|v\|_{Q(x|i| 0)}^{2}+C_{1}\|v\|_{Q^{0}(x)}^{2}+\|f\|_{Q^{0}(x)}^{2} \text {, }
$$

where $C_{1}$ is a constant, depending only on the quantities that $C\left(h_{0}\right)$ is allowed to depend on.

Now (3.9) implies that

$$
\begin{aligned}
\|v\|_{Q\left(x|i| x_{i}\right)}^{2} \leqq & C_{1} \sum_{i=1}^{n}\|v\|_{Q x(|i| 0)}^{2}+\|f\|_{Q^{0}(x)}^{2} \\
& +C_{1} \sum_{i=0}^{k_{i}}\left[h^{n-1} \sum_{Q_{h}(x|i| \& h)}|v|^{2}\right] h,
\end{aligned}
$$


where $k_{i} h=x_{i}$. It can be shown that (cf. Lees $[2$, p. 170]) (3.10) has as a consequence

$$
\|v\|_{Q\left(x|i| x_{i}\right)}^{2} \leqq 2 e^{2 C_{1} x_{i}}\left[C_{1} \sum_{i=1}^{n}\|v\|_{Q(x|i| 0)}^{2}+\|f\|_{Q^{0}(x)}^{2}\right]
$$

Finally, if we sum (3.11) with respect to the index $i$, we get the desired inequality (3.1).

The energy inequality we have just established has an important practical application as an immediate consequence; the solution of the finite-difference problem (2.1), (2.2) is stable under small changes in the data. This is also true of the semi-linear problem.

An equally important consequence of the energy inequality is the following approximation theorem.

THEOREM 3. If $u(x)$ is a smooth solution of the initial value problem (1.1), (1.2) in $Q\left(x^{0}\right), x^{0} \in Q_{h}$, and $v_{h}(x)$ is the solution of the finitedifference problem (2.1), (2.2) then

$$
E_{x^{0}}(u)=\max _{\boldsymbol{y} \in Q_{h}\left(x^{0}\right)} \sum_{i=1}^{n}\left\|u-v_{h}\right\|_{Q\left(y|i| y_{i}\right)}^{2}
$$

approaches zero as $h \rightarrow 0$. If, in addition, $u \in C^{2}\left(Q\left(x^{0}\right)\right)$, then $E_{x^{0}}(u)$ $=O\left(h^{2}\right)$.

Proof. Apply the energy inequality to the difference $u-v_{h}$ (cf. [3]).

Theorem 3 also holds for the semi-linear problem. However an important practical advantage can be obtained if we approximate (1.3) by

$$
\sum_{i=1}^{n} A^{i}(x) v_{x_{i}}=F\left(x, \bar{E}_{j}[v]\right) .
$$

This simple device avoids the necessity of employing a time consuming iterative procedure for solving the system

$$
\sum_{i=1}^{n} A^{i}(x) v_{\bar{x}_{i}}=F(x, v)
$$

since the system (3.13) is actually linear. And, what is more important, this device leaves the stability and convergence properties unaffected; Theorem 3 holds verbatim for (3.13).

- It is at this point that the mesh size must be restricted; the inequality holds if $C_{1} h \leqq 1 / 2$. 
4. Additional estimates. In this section we derive a priori estimates for the higher order difference quotients of the solution of (2.1), (2.2). Such estimates are needed for the existence theorem given in the next section.

In the remainder of this paper we assume, for simplicity, that $B=0$ in $(2.1)$; no generality is gained by keeping this term.

TheOREM 4. Let $x \in Q_{h}$ and let the functions $A^{i}, g^{i}$ and $f$ be of class $C^{1}$. If $v$ is a solution of (2.1), (2.2), then there exist positive constants $h_{0}$ and $M$, not depending on $h$, such that

$$
\sum_{i=1}^{n}\left\|v_{x_{i}}\right\|_{Q(x)}^{2} \leqq M
$$

for $h \leqq h_{0}$.

Here $M$ depends, of course, on $A^{i}, g^{i}, f$, their first derivatives, $n$ and $x$.

Proof. We introduce the functions $w^{i}=v_{x_{i}}$. If we difference equation (2.1) with respect to $x_{j}$, we get

$$
\sum_{i=1}^{n} E_{j}\left[A^{i}\right] w_{\hat{x}_{i}}^{j}+\sum_{i=1}^{n} A_{x_{j}}^{i} E_{i}\left[w^{i}\right]=f_{x_{j}} .
$$

Also

$$
\begin{gathered}
w^{j}=g_{x_{j}}^{i} \text { on } Q_{h}(\infty|i| 0) \quad \text { for } i \neq j, \\
\bar{E}_{i} w^{i}=-\left(A^{i}\right)^{-1} \sum_{k \neq i}^{n} A^{k} g_{\bar{x}_{k}}^{k} \text { on } Q_{h}(\infty|i| 0) .
\end{gathered}
$$

The energy inequality can be applied to the system (4.2), (4.3), and we get the inequality

$$
\begin{aligned}
\sum_{i=1}^{n}\left\|w^{j}\right\|_{Q\left(x|i| x_{i}\right)}^{2} \leqq & C \sum_{i=1}^{n}\left\|w^{j}\right\|_{Q(x|i| 0)}^{2}+C\left\|f_{x_{j}}\right\|_{Q^{0}(x)}^{2} \\
& +C \sum_{j=1}^{n}\left\|w^{j}\right\|_{Q(x)}^{2} .
\end{aligned}
$$

Let $z=\sum_{j=1}^{n}\left[w^{j}\right]^{2}$. Then (4.4) implies that

$$
\begin{aligned}
\sum_{i=1}^{n}\|z\|_{Q\left(x|i| x_{i}\right)}^{2} \leqq & C \sum_{i=1}^{n}\|z\|_{Q(x|i| 0)}^{2}+C \sum_{j=1}^{n}\left\|f_{x_{j}}\right\|_{Q^{0}(x)}^{2} \\
& +C n\|z\|_{Q(x)}^{2} .
\end{aligned}
$$


Since the first two terms on the right hand side of (4.5) are bounded, independent of $h$, we conclude, as in the proof of Theorem 3, that

$$
\|z\|_{Q(x)}^{2} \leqq M,
$$

which is the desired result.

Repeating this argument, we obtain the

THEOREM 5. Let $x \in Q_{h}$ and let the functions $A^{i}, g^{i}$ and $f$ be of class $C^{*}$. If $v$ is a solution of (2.1), (2.2), then there exist positive constants $h_{0}$ and $M_{s}$, independent of $h$, such that

$$
\left\|D^{k_{v}}\right\|_{Q(x)} \leqq M_{k} ; \quad(0 \leqq k \leqq s) ; \quad h \leqq h_{0},
$$

where $D^{k_{v}}$ denotes any kth order forward difference quotient of $v$.

\section{Existence and uniqueness.}

Theorem 6. If the functions $A^{i}, g^{i}$ and $f$ are of class $C^{n+2}$, then there exists a unique solution of (1.1), (1.2) which is of class $C^{1}$.

Proof. Let $e=(1,1, \cdots, 1)$. We shall construct the solution in $\bar{Q}(e)$. Consider the sequence of meshes $\left\{Q_{k}\right\}$ where $h_{k}=2^{-k}$, $(k=0,1, \cdots)$. The set

$$
D=\bigcup_{k=0}^{\infty} \bar{Q}_{k}(e)
$$

is everywhere dense in $\bar{Q}(e)$. Let $v_{k}$ denote the solution of the finitedifference problem (2.1), (2.2) over $\bar{Q}_{k}$.

We extend the function $v_{k}$ to all of $\bar{Q}(e)$ by linear interpolation. The extended function, which we still denote by $v_{k}$, is continuous on $\bar{Q}(e)$.

We have

$$
v_{k}\left(x_{1}, \cdots, x_{n}\right)=v_{k}\left(0, x_{2}, \cdots, x_{n}\right)+2^{-k} \sum_{y=0}^{x_{1}-2^{-k}} v_{k, x_{1}}\left(y, x_{2}, \cdots, x_{n}\right) .
$$

Hence, by Schwarz' inequality

$$
\begin{aligned}
& \left|v_{k}\left(x_{1}, \cdots, x_{n}\right)\right|^{2} \\
& \quad \leqq 2\left|v_{k}\left(0, x_{2}, \cdots, x_{n}\right)\right|^{2}+2^{-k+1} \sum_{y=0}^{1}\left|v_{k, x_{1}}\left(y, x_{2}, \cdots, x_{n}\right)\right|^{2} .
\end{aligned}
$$

If we apply the same reasoning to $v_{k, x_{1}}$, except that now we operate with the second variable $x_{2}$, we get 


$$
\begin{aligned}
& \left|v_{k}\left(x_{1}, \cdots, x_{n}\right)\right|^{2} \\
& \leqq \\
& \quad 2\left|v_{k}\left(0, x_{2}, \cdots, x_{n}\right)\right|^{2}+2^{2-k} \sum_{y=0}^{1}\left|v_{k, x_{1}}\left(y, 0, \cdots, x_{n}\right)\right|^{2} \\
& \quad+2^{2-k} \sum_{y, z=0}^{1}\left|v_{k, x_{1} x_{2}}\left(y, z, x_{3}, \cdots, x_{n}\right)\right|^{2} .
\end{aligned}
$$

Continuing in this way, we find that $\left|v_{k}\right|$ can be estimated in terms of bounds for the initial data, their derivatives up to the order $n-1$ and the constant $M_{n}$ of Theorem 5 .

Since linear interpolation does not increase the maximum of $v_{k}$, it follows that its linear extension is uniformly bounded on $\bar{Q}(e)$. Similarly the linear extensions of the forward difference quotients of $v_{k}$, up to the order two, are likewise uniformly bounded on $\bar{Q}(e)$. Therefore, since linear interpolation does not increase the Lipschitz constant of $v_{k}$, the sequences $\left\{v_{k}\right\}$ and $\left\{v_{k, x_{i}}\right\}$ are uniformly bounded and equicontinuous. By Ascoli's theorem, there is a subsequence of the sequence $\left\{v_{k}\right\}$ which converges uniformly on $\bar{Q}(e)$ to a continuous function $u(x)$. It is easy to show that a subsequence of $\left\{v_{k, x_{i}}\right\}$ converges uniformly on $\bar{Q}(e)$ to a continuous function $w_{i}(x)$, and that $w_{i}(x)=\partial u / \partial x_{i}$. Clearly we can arrange things so that the same subsequence of the sequences $\left\{v_{k}\right\},\left\{v_{k, x_{i}}\right\}$ converge uniformly.

It is immediate that $u$ is a solution of (1.1), (1.2) at each point of the dense set $D$. By continuity, $u$ satisfies (1.1), (1.2) everywhere in $\bar{Q}(e)$. This proves the existence part of the theorem.

The uniqueness part of the theorem follows from the energy inequality in [3].

REMARKS. (1) Since the sequence $\left\{v_{k}\right\}$ has the property that every subsequence has a further subsequence converging uniformly to a unique, continuous limit function, the original sequence must also converge uniformly to this limit function. Thus, $v_{k}$ converges uniformly to $u$ on $\bar{Q}(e)$.

(2) This theorem also holds for the semi-linear equation, provided that $F(x, u)$ is smooth enough.

\section{REFERENCES}

1. K. O. Friedrichs, Symmetric hyperbolic linear differential equations, Comm. Pure Appl. Math. vol. 7 (1954) pp. 345-392.

2. Milton Lees, Approximate solutions of parabolic equations, J. Indust. Appl. Math. vol. 7 (1959) pp. 167-183.

3. Burton Wendroff, Finite-difference approximations to solutions of partial differential equations, Los Alamos Scientific Laboratory Report LA-2157, Los Alamos, New Mexico, December, 1957.

The Institute for Advanced Study and

BrookhaVEN NATIONAL Laboratory 